\title{
ON THE STUDY OF DOUBLE FOURIER SERIES BY DOUBLE MATRIX SUMMABILITY METHOD
}

\author{
SHYAM LAL AND VIRENDRA NATH TRIPATHI
}

\begin{abstract}
In this paper a new theorem on double matrix summability of double Fourier series has been established. This theorem is a generalization of several known and unknown results.
\end{abstract}

\section{Introduction}

Harmonic and $\left(N, p_{n}\right)$ summability of single Fourier series have been studied by a number of researchers like lyengar (1943), Siddiqui (1948), Pati (1961), Singh (1963), Hirokawa (1968) and Izumi and Izumi (1968). In 1953, Chow for the first time studied Cesàro summability of double Fourier series. In 1958, Sharma extended the result of Chow for $(H, 1,1)$ summability which is weaker than $(C, 1,1)$ summability of double Fourier series. Working in the same direction in 1932, Hille and Tamarkin defined double Nörlund summability $\left(N, p_{m}, q_{n}\right)$ of double Fourier series. After this double Nörlund summability of double Fourier series was studied by several of researchers like Tripathi and Singh (1981), Tripathi and Ojha (1995), Tripathi and Lal (1984), Mishra (1985), Lal (1992), Singh, Lal and Singh (1995) and Lal and Verma (1998). But nothing seems to have been done so far to study double Fourier series by a double factorable summability method which, as known, includes as special cases, the methods of $(C, 1,1),(H, 1,1)$ and $\left(N, p_{m}, q_{n}\right)$. In this paper a more general result than those of Chow (1953), Lal (1992) and Singh, Lal and Singh (1995) have been established, which include their results as particular cases.

\section{Definitions and Notations}

Let $f(u, v)$ be a function of $(u, v)$, periodic with respect to $u$ and with respect to $v$, in each case, with period $2 \pi$, and summable in the square $Q(-\pi,-\pi, \pi, \pi)$. The double Fourier series of a function $f(u, v)$ is

$\sum_{m=0}^{\infty} \sum_{n=0}^{\infty} \lambda_{m n}\left[a_{m n} \cos \mathrm{mu} \cos \mathrm{nv}+b_{m n} \mathrm{mu} \cos \mathrm{nv}+C_{m n} \cos \mathrm{mu} \sin \mathrm{nv}+d_{m n} \sin \mathrm{mu} \sin \mathrm{nv}\right]$

Received September 30, 2000; revised August 6, 2001.

2000 Mathematics Subject Classification. 42B05, 42B08.

Key words and phrases. Double Fourier series $\left(N, p_{m}, q_{n}\right)$ means, $(H, 1,1),(C, 1,1)$ summabilities and double matrix summability. 
$=\sum_{m=0}^{\infty} \sum_{n=0}^{\infty} \lambda_{m n} A_{m n(u, v)}$

where

$$
\lambda_{m n}=\left\{\begin{array}{lll}
1 / 4 & \text { for } m=0, \quad n=(\text { or } m=n=0) \\
1 / 2 & \text { for } m>0, \quad n>0 \text { and } m=0, \quad n>0 \\
1 & \text { for } m>0, \quad n>0 .
\end{array}\right.
$$

and $a_{m, n}=\frac{1}{\pi^{2}} \iint_{Q} f(u, v) \cos$ mu con nv $d u d v$, with similar three similar expressions for $b_{m n}, c_{m n}$ and $d_{m n}$, where $Q$ denotes the fundamental square $(-\pi, \pi) \times(-\pi, \pi)$. The double series $\sum_{m=0}^{\infty} \sum_{n=0}^{\infty} a_{m n}$ with the sequence of $(m, n)^{\text {th }}$ parital sums $\left\{S_{m n}\right\}$ is said to be summable by a double matrix summability method or summable $(T, S)$ if $t_{m, n}$ tends to a limit $s$ as $(m, n) \rightarrow \infty$, where the double matrix mean $t_{m n}$ is given by

$$
t_{m, n}=\sum_{i=0}^{m} \sum_{k=0}^{n} a_{m, i} b_{n, k} s_{i, k}=\sum_{i=0}^{m} \sum_{k=0}^{n} a_{m, m-i} b_{n, n-k} s_{m-i, n-k}
$$

The regularity conditions of double matrix summability means are given by

$$
\begin{aligned}
& \sum_{i=0}^{m} \sum_{k=0}^{n} a_{m, i} b_{n, k} \rightarrow 1 \quad \text { as }(m, n) \rightarrow \infty \\
& \lim _{m, n} \sum_{k=0}^{n}\left|a_{m i} b_{n k}\right|=0, \quad \text { for each } \quad i=1,2, \ldots \\
& \lim _{m, n} \sum_{i=0}^{n}\left|a_{m i} b_{n k}\right|=0, \quad \text { for each } \quad k=1,2, \ldots
\end{aligned}
$$

Three important particular cases of the double matrix summability method are given by:

(i) $(C, 1,1)$ summability mean [Chow (1953)] if $a_{m, i}=\frac{1}{m+1} \forall m$ and $b_{n, k}=\frac{1}{n+1} \forall n$

(ii) $(H, 1,1)$ summability mean [Sharma (1958)] if $a_{m, i}=\frac{1}{(m-i+1) \log m}$ and $b_{n, k}=$ $\frac{1}{(n-k+1) \log n}$

(iii) $\left(N, p_{m}, q_{n}\right)$ summability mean [Hille and Tamarkin (1932)] if $a_{m, i}=\frac{p_{m-i}}{P_{m}}$ and $b_{n, k}=\frac{q_{n-k}}{Q_{n}}$ provided $P_{m}=\sum_{i=0}^{m} p_{i}$ and $Q_{n}=\sum_{k=0}^{n} q_{k}$.

We write

$$
\begin{aligned}
\phi(u, v)= & \phi(x, y ; u, v) \\
= & 1 / 4[f(x+u, y+v)+f(x+u, y-v)+f(x-u, y+v) \\
& +f(x-u, y-v)-4 f(x, y)]
\end{aligned}
$$


and

$$
\begin{aligned}
\phi(u, v) & =\int_{0}^{u} \int_{0}^{v}|\phi(s, t)| d s d t \\
\phi_{1}(u, t) & =\int_{0}^{u}|\phi(s, t)| d s \\
\phi_{2}(s, v) & =\int_{0}^{v}|\phi(s, t)| d t \\
\tau=\left(\frac{1}{t}\right) & =\text { integral part of } \frac{1}{t} \\
\sigma=\left(\frac{1}{s}\right) & =\text { integral part of } \frac{1}{s}
\end{aligned}
$$

$$
\begin{aligned}
K_{m}(s) & =\frac{1}{2 \pi} \sum_{i=0}^{m} a_{m, m-i} \frac{\sin \left(m-i+\frac{1}{2}\right) s}{\sin \frac{1}{2} s} \\
K_{n}(t) & =\frac{1}{2 \pi} \sum_{k=0}^{n} b_{n, n-k} \frac{\sin \left(n-k+\frac{1}{2}\right) t}{\sin \frac{1}{2} t}
\end{aligned}
$$

\section{Main Theorem}

Much is known for the factorable $(C, 1,1),(H, 1,1)$ and $\left(N, p_{m}, q_{n}\right)$ summabilities of double Fourier series. But till now no work seems to have been done on double matrix summability of double Fourier series. The object of this theorem is to extend the results of Chow (1953), Sharma (1958), Tripathi and Singh (1981) and Tripathi and Ojha (1982), Mishra (1985), Lal (1992) and Singh, Lal and Singh (1995) on $(C, 1,1)$, $(H, 1,1)$ and double Nörlund summability method to a more general class of double matrix summability of double Fourier series in two ways. In fact we shall establish the following theorem.

Theorem. Let $\|T\|=\left(a_{m, j}\right)$ and $\|S\|=\left(b_{n, k}\right)$ be two inifinite triangular matrices with $a_{m, i} \geq 0, b_{n, k} \geq 0$. $A_{m \tau}=\sum_{i=0}^{\tau} a_{m, m-i}, B_{n, \eta}=\sum_{k=0}^{\eta} b_{n, n-k}, A_{m m}=1$ for each $m \geq 0$ and $B_{n, n}=1 \forall n \geq 0$. Let $\left\{a_{m, i}\right\}_{i=0}^{m}$ and $\left\{b_{n, k}\right\}_{k=0}^{n}$ be two real non-negative and non-decreasing sequences with respect to $i$ and $k$, respectively. Assume that $(T, S)$ is regular.

If the conditions

$$
\begin{aligned}
\Phi|u, v|= & \int_{0}^{u} \int_{0}^{v}|\Phi(s, t)| d s d t=o\left(\frac{\alpha\left(\frac{1}{u}\right) u}{\log \frac{1}{u}} \frac{\beta\left(\frac{1}{v}\right) v}{\log \frac{1}{v}}\right) \quad \text { as }(u, v) \rightarrow 0 \\
& \int_{0}^{\pi} \phi_{1}(u, t) d t=o\left(\frac{\alpha\left(\frac{1}{u}\right) u}{\log \frac{1}{u}}\right)
\end{aligned}
$$

and

$$
\int_{0}^{\pi} \phi_{2}(s, v) d s=o\left(\frac{\beta\left(\frac{1}{v}\right) v}{\log \frac{1}{v}}\right)
$$


hold then the double Fourier series (2.1) is double matrix summable to $f(x, y)$, at the point $(u, v)=(x, y)$, provided $\alpha(t)$ and $\beta(t)$ are two positive monotonic non-increasing fucntions such that

$$
\begin{aligned}
\int_{1}^{m} \frac{\alpha(x) A_{m, x}}{x \log x} d x & =O(1) \\
\int_{1}^{n} \frac{\beta(y) B_{n, y}}{y \log y} d y & =O(1),
\end{aligned}
$$

and $\frac{\alpha\left(\frac{1}{s}\right)}{\log \left(\frac{1}{s}\right)}, \frac{\beta\left(\frac{1}{t}\right)}{\log \left(\frac{1}{t}\right)}$ are bounded functions in $\left[\frac{1}{m}, \delta\right]$ and $\left[\frac{1}{n}, \xi\right]$ respectively.

\section{Lemmas}

For the proof of our theorem the following lemmas are required.

Lemma 4.1.[Lal and Pratap (1999)] Let $K_{m}(s)$ and $K_{n}(t)$ be given by $(2.2)$ and (2.3) respectively then

(i) $K_{m}(s)=O(m)$ for $0 \leq s \leq(1 / m)$

(ii) $K_{n}(t)=O(n)$ for $0 \leq t \leq(1 / n)$

Lemma 4.2. [Lal (2000)] (i) If $\left(a_{m, \mu}\right)$ is non-negative and non-decreasing with $\mu$ then for $0 \leq a \leq b \leq \infty$ and $0 \leq s \leq \pi$ and any $m$.

$$
\left|\sum_{\mu=a}^{b} a_{m, m-\mu} e^{i(m-\mu) s}\right| \leq O\left(A_{m, \sigma}\right)
$$

where $\sigma=$ integral part of $\left(\frac{1}{s}\right)=\left[\frac{1}{s}\right]$

Note. In the proof of this lemma Lal [14] has also shown that

$$
\frac{a_{m, m-\sigma}}{s}=O\left(A_{m, \sigma}\right)
$$

Similarly,

$$
\frac{b_{n, n-\tau}}{t}=O\left(B_{n, \tau}\right)
$$

(ii) If $\left(b_{n, \nu}\right)$ is non-negative and non-decreasing with $\nu$ then for $0 \leq a \leq b \leq \infty, 0 \leq t \leq \pi$ and any $n$

$$
\left|\sum_{\nu=a}^{h} b_{n, n-\nu} e^{i(n-\nu) t}\right| \leq O\left(B_{n, \tau}\right)
$$

where $\tau=$ integral part of $\left(\frac{1}{t}\right)$

Lemma 4.3. Let $K_{m}(s)$ be given by (2.3), then under the condition of our theorem on $\left(a_{m, \mu}\right)$

$$
K_{m}(s)=O\left(\frac{A_{m, \sigma}}{s}\right), \quad \text { for } 0<\frac{1}{m} \leq s \leq \pi
$$


Proof. Since $0<\frac{1}{m} \leq S \leq \pi, \sin \frac{s}{2}>\frac{s}{\pi}$, therefore for $t>0$ and $s \leq m$. We have

$$
\begin{aligned}
\left|K_{m}(s)\right| & =\left|\frac{1}{2 \pi} \sum_{\mu=0}^{m} a_{m, m-\mu} \frac{\sin \left(m-\mu-\frac{1}{2}\right) s}{\sin \frac{s}{2}}\right| \\
& \leq \frac{1}{2 \pi} \mid \text { Imaginary part of } \sum_{\mu=0}^{m} a_{m, m-\mu} \frac{e^{i\left(m-\mu-\frac{1}{2}\right) s}}{\sin \frac{s}{2}} \mid \\
& =O\left(\frac{1}{s}\right)\left|\sum_{\mu=0}^{m} a_{m, m-\mu} e^{i(m-\mu) s}\right|\left|e^{-i \cdot \frac{s}{2}}\right| \\
& =O\left(\frac{1}{s}\right)\left|\sum_{\mu=0}^{m} a_{m, m-\mu} e^{i(m-\mu) s}\right| \\
& =O\left(\frac{A_{m, \sigma}}{s}\right) \text { by lemma (4.2.1) }
\end{aligned}
$$

Lemma 4.4. Let $K_{n}(t)$ by given by (2.4) then under the condition of our theorem $\left(b_{n, \nu}\right)$,

$$
K_{n}(t)=O\left(\frac{B_{n, \tau}}{t}\right), \quad \text { for } 0<\frac{1}{n} \leq t \leq \pi
$$

Proof. It can be proved similar to lemma (4.3).

\section{Proof of the Main Theorem}

$(i, k)^{\text {th }}$ partial sum of the series $(2.1)$ at $(u, v)=(x, y)$ is given by

$$
s_{i, k}-f(x, y)=\frac{1}{4 \pi^{2}}\left(\int_{0}^{\pi} \int_{0}^{\pi} \phi(s, t) \frac{\sin \left(i+\frac{1}{2}\right) s}{\sin \frac{s}{2}} \frac{\sin \left(k+\frac{1}{2}\right) t}{\sin \frac{t}{2}}\right) d s d t
$$

Then

$$
\sum_{i=0}^{m} \sum_{k=0}^{n} a_{m, i} b_{n, k}\left\{s_{i, k}-f(x, y)\right\}=\sum_{i=0}^{m} \sum_{k=0}^{n} a_{m, m-i} b_{n, n-k}\left\{s_{m-i, n-k}-f(x, y)\right\}
$$

or

$$
\begin{aligned}
t_{m, n}-f(x, y) & =\int_{0}^{\pi} \int_{0}^{\pi} \phi(s, t) K_{m}(s) K_{n}(t) d s d t \\
& =\left(\int_{0}^{\delta} \int_{0}^{\xi}+\int_{0}^{\delta} \int_{\xi}^{\pi}+\int_{\delta}^{\pi} \int_{0}^{\xi}+\int_{\delta}^{\pi} \int_{\xi}^{\pi}\right) \phi(s, t) K_{m}(s) K_{n}(t) d s d t \\
& \equiv I_{1}+I_{2}+I_{3}+I_{4}, \quad \text { say }
\end{aligned}
$$


Now, considering

$$
\begin{aligned}
I_{1} & =\left(\int_{0}^{\delta} \int_{0}^{\xi}\right) \phi(s, t) K_{m}(s) K_{n}(t) d s d t \\
& =\left(\int_{0}^{\frac{1}{m}} \int_{0}^{\frac{1}{n}}+\int_{\frac{1}{m}}^{\delta} \int_{0}^{\frac{1}{n}}+\int_{0}^{\frac{1}{m}} \int_{\frac{1}{n}}^{\xi}+\int_{\frac{1}{m}}^{\delta} \int_{\frac{1}{n}}^{\xi}\right) \phi(s, t) K_{m}(s) K_{n}(t) d s d t \\
& =I_{1.1}+I_{1.2}+I_{1.3}+I_{1.4}, \quad \text { say }
\end{aligned}
$$

where, for $s \leq \delta, t \leq \xi$, (3.1) hold

Now, let us consider

$$
\begin{aligned}
I_{1.1} & =O(m n) \int_{0}^{\frac{1}{m}} \int_{0}^{\frac{1}{n}}|\phi(s, t)| d s d t \\
& =O(m n) o\left(\frac{\alpha(m) \beta(n)}{m n \log (m) \log (n)}\right) \text { by } \\
& =o\left(\frac{\alpha(m) \beta(n)}{\log m \log n}\right) \\
& =o(1),
\end{aligned}
$$

as $(m, n) \rightarrow \infty\left(\because \frac{\alpha(m)}{\log m}=o(1)\right.$ as $m \rightarrow \infty$, as $\alpha(t)$ is a monotonic decreasing function of $t$, and similarly $\frac{\beta(n)}{\log n}=o(1)$ as $\left.n \rightarrow \infty\right)$.

Now

$$
\begin{aligned}
I_{1.2}= & \left(\int_{\frac{1}{m}}^{\delta} \int_{0}^{\frac{1}{n}}\right) \phi(s, t) K_{m}(s) K_{n}(t) d s d t \\
\leq & \left(\int_{0}^{\frac{1}{n}}\left|K_{n}(t)\right| d t \int_{\frac{1}{m}}^{\delta}|\phi(s, t)| \frac{A_{m,[1 / s]}}{s} d s\right) \text { by lemma }(4.3) \\
= & \left(\int_{0}^{\frac{1}{n}} O(n) d t \int_{\frac{1}{m}}^{\delta}|\phi(s, t)| \frac{A_{m,[1 / s]}}{s} d s\right) \text { by lemma }(4.1) \\
= & O(n)\left(\int_{0}^{\frac{1}{n}} d t \int_{\frac{1}{m}}^{\delta}|\phi(s, t)| \frac{A_{m,[1 / s]}}{s} d s\right) \\
= & O(n)\left(\int_{0}^{\frac{1}{n}} d t\left\{\left(\phi_{1}(s, t) \frac{A_{m,[1 / s]}}{s}\right)_{\frac{1}{m}}^{\delta} \int_{\frac{1}{m}}^{\delta} \phi_{1}(s, t) \frac{d}{d s}\left(\frac{A_{m,[1 / s]}}{s}\right) d s\right\}\right) \\
= & O(n)\left(\int_{0}^{\frac{1}{n}} \phi_{1}(\delta, t) \frac{A_{m,[1 / s]}}{\delta} d t\right)+O(n) m A_{m, m} \int_{0}^{\frac{1}{n}} \phi_{1}\left(\frac{1}{m}, t\right) d t \\
& +O(n)\left(\int_{0}^{\frac{1}{n}} d t \int_{\frac{1}{m}}^{\delta}\left|\phi_{1}(s, t)\right| \frac{d}{d s}\left(\frac{A_{m,[1 / s]}}{s}\right) d s\right) \\
= & I_{1.2 .1}+I_{1.2 .2}+I_{1.2 .3}, \operatorname{say}
\end{aligned}
$$


Now

$$
\begin{aligned}
I_{1.2 .1} & =O(n) \int_{0}^{\frac{1}{n}} \Phi_{1}(\delta, t) d t \\
& =O(n) \Phi\left(\delta, \frac{1}{n}\right) \\
& =O(n) o\left(\frac{\alpha\left(\frac{1}{\delta}\right) \delta}{\log \frac{1}{\delta}} \cdot \frac{\beta(n) \frac{1}{n}}{\log n}\right) \\
& =o\left(\frac{\beta(n)}{\log n}\right) \\
& =o(1), \text { as }(m, n) \rightarrow \infty, \text { by hypothesis of the theorem. }
\end{aligned}
$$

Next

$$
\begin{aligned}
I_{1.2 .2} & =O(m n) \int_{0}^{\frac{1}{n}} \Phi_{1}\left(\frac{1}{m}, t\right) d t \\
& =O(m n) \Phi\left(\frac{1}{m}, 1 / n\right) \\
& =O(m n) o\left(\frac{\alpha(m)}{m \log m} \cdot \frac{\beta(n)}{n \log n}\right) \\
& =o\left(\frac{\alpha(m)}{\log m} \cdot \frac{\beta(n)}{\log n}\right) \\
& =o(1), \text { as }(m, n) \rightarrow \infty, \text { by hypothesis of the theorem. }
\end{aligned}
$$

Lastly

$$
\begin{aligned}
I_{1.2 .3} & =O(n) \int_{0}^{\frac{1}{n}} d t \int_{\frac{1}{m}}^{\delta} \Phi_{1}(s, t) \frac{A_{m,[1 / s]}}{s^{2}} d s+O(n) \int_{0}^{\frac{1}{n}} d t \int_{\frac{1}{m}}^{\delta} \frac{\Phi_{1}(s, t)}{s} \frac{d}{d s}\left(A_{m,[1 / s]}\right) d s \\
& =I_{1.2 .3 .1}+I_{1.2 .3 .2}
\end{aligned}
$$

Now

$$
\begin{aligned}
I_{1.2 .3 .1} & =O(n) \int_{0}^{\frac{1}{n}} d t \int_{\frac{1}{m}}^{\delta} \Phi_{1}(s, t) \frac{A_{m,[1 / s]}}{s^{2}} d s \\
& \leq O(n) \int_{\frac{1}{m}}^{\delta}\left\{\int_{0}^{\frac{1}{n}} \Phi_{1}(s, t) d t\right\} \frac{A_{m,[1 / s]}}{s^{2}} d s \\
& =O(n) \int_{\frac{1}{m}}^{\delta} \Phi\left(s, \frac{1}{n}\right) \frac{A_{m,[1 / s]}}{s^{2}} d s \\
& =O(n) \int_{\frac{1}{m}}^{\delta} o\left(\frac{\alpha\left(\frac{1}{s}\right) s}{\log \frac{1}{s}} \cdot \frac{\beta(n)}{n \log n}\right) \frac{A_{m,[1 / s]}}{s^{2}} d s \\
& =o\left(\frac{\beta(n)}{\log n}\right) \int_{\frac{1}{m}}^{\delta} \frac{\alpha\left(\frac{1}{s}\right) A_{m,[1 / s]}}{s \log [1 / s]} d s
\end{aligned}
$$




$$
\begin{aligned}
& =o\left(\frac{\beta(n)}{\log n}\right) \int_{\frac{1}{\delta}}^{m} \frac{\alpha(x) A_{m, x}}{x \log x} d x \\
& =o\left(\frac{\beta(n)}{\log n}\right) \int_{1}^{m} \frac{\alpha(x) A_{m, x}}{x \log x} d x \\
& =o\left(\frac{\beta(n)}{\log n}\right) O(1) \quad \text { by }(3.4) \\
& =o(1), \quad \text { as }(m, n) \rightarrow \infty .
\end{aligned}
$$

Nextly,

$$
\begin{aligned}
I_{1.2 .3 .2} & =O(n) \int_{0}^{\frac{1}{n}} d t \int_{\frac{1}{m}}^{\delta} \frac{\Phi_{1}(s, t)}{s} \frac{d}{d s}\left(A_{m,[1 / s]}\right) d s \\
& \leq O(n) \int_{\frac{1}{m}}^{\delta}\left\{\int_{0}^{\frac{1}{n}} \Phi_{1}(s, t) d t\right\} \frac{1}{s} \frac{d}{d s}\left(A_{m,[1 / s]}\right) d s \\
& =O(n) \int_{\frac{1}{m}}^{\delta} \Phi(s, 1 / n) \frac{1}{s} \frac{d}{d s}\left(A_{m,[1 / s]}\right) d s \\
& =O(n) \int_{\frac{1}{m}}^{\delta} o\left(\frac{\alpha\left(\frac{1}{s}\right) s}{\log \frac{1}{s}} \cdot \frac{\beta(n)}{n \log n}\right) \frac{d}{d s}\left(A_{m,[1 / s]}\right) d s \\
& =o\left(\frac{\beta(n)}{\log n}\right) \int_{m}^{\frac{1}{\delta}} \frac{\alpha(x)}{\log x} \frac{d}{d x}\left(A_{m,[1 / x]}\right) d x \\
& =o\left(\frac{\beta(n)}{\log n}\right) \int_{m}^{\frac{1}{\delta}} O(1) \frac{d}{d x}\left(A_{m x}\right) d x \\
& =o\left(\frac{\beta(n)}{\log n}\right)\left|A_{m x}\right| \frac{1}{\delta} \\
& =o\left(\frac{\beta(n)}{\log n}\right) O(1)=o(1) .
\end{aligned}
$$

Thus,

$$
I_{1.2}=o(1) \text { as }(m, n) \rightarrow \infty .
$$

Similarly $I_{1.3}=o(1)$ as $(m, n) \rightarrow \infty$.

Now,

$$
\begin{aligned}
I_{1.4}= & \left(\int_{\frac{1}{m}}^{\delta} \int_{\frac{1}{n}}^{\xi}|\phi(s, t)| \frac{A_{m,[1 / s]}}{s} \cdot \frac{B_{n \cdot[1 / t]}}{t} d t d s\right) \\
= & O\left(\frac{B_{n,[1 / \xi]} A_{m,[1 / \delta]} \Phi(\delta, \xi)}{\delta \xi}-\frac{m B_{n,[1 / \xi]} A_{m m} \Phi(1 / m, \xi)}{\xi}\right. \\
& -\frac{B_{n,[1 / \xi]}}{\xi} \cdot \int_{\frac{1}{m}}^{\delta} \Phi(s, \xi) \frac{d}{d s}\left(\frac{A_{m,[1 / s]}}{s}\right) d s
\end{aligned}
$$




$$
\begin{aligned}
& -\frac{n B_{n n} A_{m,[1 / \delta]} \Phi(\delta, 1 / n)}{\delta}+m n B_{n n} A_{m m} \Phi(1 / m, 1 / n) \\
& +n B_{n n} \int_{\frac{1}{m}}^{\delta} \Phi(s, 1 / n) \frac{d}{d s}\left(\frac{A_{m,[1 / s]}}{s}\right) d s \\
& +\frac{A_{m,[1 / \delta]}}{\delta} \int_{\frac{1}{n}}^{\delta} \Phi_{2}(s, t) \frac{d}{d t}\left(\frac{B_{n,[1 / t]}}{t}\right) d t-m A_{m m} \int_{\frac{1}{n}}^{\xi} \Phi_{2}(1 / m, t) \frac{d}{d t}\left(\frac{B_{n,[1 / t]}}{t}\right) d t \\
& \left.-\int_{\frac{1}{m}}^{\delta} \int_{\frac{1}{n}}^{\xi} \Phi(s, t) \frac{d}{d s}\left(\frac{A_{m,[1 / s]}}{s}\right) \frac{d}{d t}\left(\frac{B_{n,[1 / t]}}{t}\right) d t d s\right) \\
& =I_{1.4 .1}+I_{1.4 .2}+I_{1.4 .3}+I_{1.4 .4}+I_{1.4 .5}+I_{1.4 .6}+I_{1.4 .7}+I_{1.4 .8}+I_{1.4 .9}, \quad \text { say }
\end{aligned}
$$

Let us consider

$$
\begin{aligned}
I_{1.4 .1} & =\frac{B_{n,[1 / \xi]} A_{m,[1 / \delta]} \Phi(\delta, \xi)}{\delta \xi} \\
& =\frac{B_{n,[1 / \xi]} A_{m,[1 / \delta]}}{\delta \xi} o\left(\frac{\alpha\left(\frac{1}{\delta}\right) \delta}{\log \left(\frac{1}{\delta}\right)} \cdot \frac{\beta\left(\frac{1}{\xi}\right) \xi}{\log \left(\frac{1}{\xi}\right)}\right) \\
& =o\left(\frac{B_{n,[1 / \xi]}}{\xi} \cdot \frac{A_{m,[1 / \delta]}}{\delta} \cdot \frac{\alpha\left(\frac{1}{\delta}\right) \delta}{\log \left(\frac{1}{\delta}\right)} \cdot \frac{\beta\left(\frac{1}{\xi}\right) \xi}{\log \left(\frac{1}{\xi}\right)}\right) \\
& =o(1), \quad a s(m, n) \rightarrow \infty, \quad \text { by the regularity of }(T, S)
\end{aligned}
$$

Nextly

$$
\begin{aligned}
I_{1.4 .2} & =\frac{-m B_{n,[1 / \xi]} A_{m m} \Phi(1 / m, \xi)}{\xi} \\
& =o\left(m, B_{n,[1 / \xi]}\left(\frac{\alpha(m)}{m \log m} \cdot \frac{\beta\left(\frac{1}{\xi}\right) \xi}{\log \xi}\right)\right) \quad\left(\because A_{m, m}=1\right) \\
& =o\left(B_{n,[1 / \xi]} \frac{\alpha(m)}{\log m} \cdot \frac{\beta\left(\frac{1}{\xi}\right) \xi}{\log \xi}\right) \\
& =o(1)\left(\frac{\alpha(m)}{\log m}\right) \\
& =o(1) \quad \text { as }(m, n) \rightarrow \infty, \quad \text { by the hypothesis of the theorem. }
\end{aligned}
$$

Now let us consider

$$
\begin{aligned}
I_{1.4 .3}= & -\frac{B_{n,[1 / \xi]}}{\xi} \int_{\frac{1}{m}}^{\delta} \Phi(s, \xi) \frac{d}{d s}\left(\frac{A_{m,[1 / s]}}{s}\right) d s \\
= & o\left(\frac{B_{n,[1 / \xi]}}{\xi}\right) \int_{\frac{1}{m}}^{\delta}\left(\frac{\alpha\left(\frac{1}{s}\right) s}{\log \frac{1}{s}} \cdot \frac{\beta\left(\frac{1}{\xi}\right) \xi}{\log \frac{1}{\xi}}\right)\left(\frac{A_{m,[1 / s]}}{s^{2}}\right) d s \\
& +o\left(\frac{B_{n \cdot[1 / s]}}{\xi}\right) \int_{\frac{1}{m}}^{\delta} o\left(\frac{\alpha\left(\frac{1}{s}\right) s}{\log \frac{1}{s}} \frac{\beta\left(\frac{1}{\xi}\right) \xi}{\log \frac{1}{\xi}}\right) \frac{1}{s} \frac{d}{d s}\left(A_{m,[1 / s]}\right) d s \\
= & I_{1.4 .3 .1}+I_{1.4 .3 .2}, \quad \text { say }
\end{aligned}
$$


Let us consider

$$
\begin{aligned}
I_{1.4 .3 .1} & =o\left(\frac{B_{n,[1 / \xi]} \beta\left(\frac{1}{\xi}\right)}{\log \frac{1}{\xi}}\right) \int_{\frac{1}{m}}^{\delta} \frac{\alpha\left(\frac{1}{s}\right)}{s \log \frac{1}{s}}\left(A_{m,[1 / s]}\right) d s \\
& =o\left(\frac{B_{n,[1 / \xi]}}{\log \frac{1}{\xi}} \beta\left(\frac{1}{\xi}\right) \frac{m \alpha(m)}{\log m} A_{m m}\right) \int_{\frac{1}{m}}^{\delta} d s \\
& =o\left(\frac{B_{n,[1 / \xi]}}{\log \frac{1}{\xi}} \beta\left(\frac{1}{\xi}\right) \frac{m \alpha(m)}{\log m} A_{m m}(1 / m)\right) \\
& =o\left(\frac{B_{n,[1 / \xi]} \beta\left(\frac{1}{\xi}\right)}{\log \frac{1}{\xi}} \frac{\alpha(m)}{\log m}\right)=o\left(B_{n,[1 / \xi]}\right) o\left(\frac{\alpha(m)}{\log m}\right) \\
& =o(1), \quad \quad \quad \quad \quad \sin (m, n) \rightarrow \infty .
\end{aligned}
$$

Further, $\quad I_{1.4 .3 .2}=O\left(\frac{B_{n,[1 / \xi]}}{\xi}\right) \int_{1 / m}^{\delta} o\left(\frac{\alpha\left(\frac{1}{s}\right) s}{\log \frac{1}{s}} \frac{\beta\left(\frac{1}{\xi}\right) \xi}{\log \frac{1}{\xi}}\right) \frac{1}{s} \frac{1}{d s}\left(A_{m,[1 / s]}\right) d s$

$$
\begin{aligned}
& =o\left(\frac{B_{n,[1 / \xi] \beta\left(\frac{1}{\xi}\right)}}{\log \frac{1}{\xi}}\right) \int_{1 / m}^{\delta} \frac{\alpha\left(\frac{1}{s}\right)}{\log \frac{1}{s}} \frac{d}{d s}\left(A_{m,[1 / s]}\right) d s \\
& =o(1) \int_{1 / m}^{\delta} O(1) \frac{d}{d s}\left(A_{m,[1 / s]}\right) d s, \quad \text { by hypothesis of the theorem } \\
& =o(1) \int_{1 / \delta}^{m} \frac{d}{d x}\left(A_{m, x}\right) d x \\
& =o(1)\left[A_{m, x}\right]_{1 / \delta}^{m} \\
& =o(1), \quad \text { as }(m, n) \rightarrow \infty .
\end{aligned}
$$

Thus we get

$$
I_{1.4 .3}=o(1), \quad \text { as }(m, n) \rightarrow \infty .
$$

Similar to $I_{1.4 .2}$

$$
I_{1.4 .4}=o(1), \quad \text { as }(m, n) \rightarrow \infty .
$$

Further

$$
\begin{aligned}
I_{1.4 .5} & =m n B_{n n} A_{m m} \Phi(1 / m, 1 / n) \\
& =o\left(m n A_{m m} B_{n n} \frac{\alpha(m)}{m \log m} \frac{\beta(n)}{n \log n}\right) \\
& =o\left(\frac{m n \alpha(m) \beta(n)}{m \log m n \log n}\right) \\
& =o(1), \quad \text { as }(m, n) \rightarrow \infty .
\end{aligned}
$$


Let us consider

$$
\begin{aligned}
I_{1.4 .6} & =n B_{n n} \int_{1 / m}^{\delta} \Phi(s, 1 / n) \frac{d}{d s}\left(\frac{A_{m,[1 / s]}}{s}\right) d s \\
& =n B_{n n} \int_{1 / m}^{\delta} \Phi(s, 1 / n) \frac{A_{m,[1 / s]}}{S^{2}} d s+n B_{n n} \int_{1 / m}^{\delta} \Phi(s, 1 / n) \frac{1}{s} \frac{d}{d s} A_{m,[1 / s]} \\
& =I_{1.4 .6 .1}+I_{1.4 .6 .2} \quad \text { say }
\end{aligned}
$$

Taking

$$
\begin{aligned}
I_{1.4 .6 .1} & =n B_{n n} \int_{1 / m}^{\delta} \Phi\left(s, \frac{1}{n}\right) \frac{A_{m,[1 / s]}}{s^{2}} d s \\
& =n B_{n n} \int_{1 / m}^{\delta} o\left(\frac{\alpha\left(\frac{1}{s}\right) s}{\log \frac{1}{s}} \frac{\beta(n)}{n \log n}\right) \cdot \frac{A_{m,[1 / s]}}{s^{2}} d s \\
& =o\left(\frac{n B_{n n} \cdot \beta(n)}{n \log n}\right) \int_{1 / m}^{\delta} \frac{\alpha\left(\frac{1}{s}\right)}{\log \frac{1}{s}} \cdot \frac{A_{m,[1 / s]}}{s} d s \\
& =o\left(\frac{\beta(n)}{\log n}\right) \int_{1 / m}^{\delta} \frac{\alpha\left(\frac{1}{s}\right)}{\log \frac{1}{s}} \frac{A_{m,[1 / s]}}{s} d s \\
& =o(1), \quad \text { as similar to } I_{1.2 .3 .1}
\end{aligned}
$$

Further

$$
\begin{aligned}
I_{1.4 .6 .2} & =n B_{n n} \int_{1 / m}^{\delta} \Phi\left(s, \frac{1}{n}\right) \frac{1}{s} \frac{d}{d s} A_{m,[1 / s]} d s \\
& =n B_{n n} \int_{1 / m}^{\delta}\left(\frac{\alpha\left(\frac{1}{s}\right) s}{\log \frac{1}{s}} \frac{\beta(n)}{n \log n}\right) \frac{1}{s} \frac{d}{d s} A_{m,[1 / s]} d s \\
& =o\left(\frac{\beta(n)}{\log n} \int_{1 / m}^{\delta} \frac{\alpha\left(\frac{1}{s}\right)}{\log \frac{1}{s}} O(1) d s\right) \\
& =o\left(\frac{\beta(n)}{\log n} \int_{m}^{1 / \delta} \frac{\alpha(x)}{\log x}\left(\frac{-d x}{x^{2}}\right)\right) \\
& =o\left(\frac{\beta(n)}{m \log n} \int_{1 / \delta}^{m} \frac{\alpha(x)}{x \log x} d x\right) \\
& =o\left(\frac{\beta(n)}{m \log n} O(1)\right)=o(1) .
\end{aligned}
$$

Thus we get

$$
I_{1.4 .6}=o(1), \quad \text { as }(m, n) \rightarrow \infty
$$

As similar to $I_{1.4 .3}$,

$$
I_{1.4 .7}=o(1), \quad \text { as }(m, n) \rightarrow \infty
$$


As similar to $I_{1.4 .6}$

$$
I_{1.4 .8}=o(1), \quad \text { as }(m, n) \rightarrow \infty
$$

Lastly

$$
\begin{aligned}
& I_{1.4 .9}=O\left(\int_{1 / m}^{\delta} \int_{1 / n}^{\xi} \Phi(s, t)\left(\frac{A_{m,[1 / s]}}{s^{2}}+\frac{1}{s} \frac{d}{d s}\left(A_{m,[1 / s]}\right)\right) \times\left(\frac{B_{n,[1 / t]}}{t^{2}}+\frac{1}{t} \frac{d}{d t}\left(B_{n,[1 / t]}\right)\right) d t d s\right) \\
& =I_{1.4 .9 .1}+I_{1.4 .9 .2}+I_{1.4 .9 .3}+I_{1.4 .9 .4} \text {. } \\
& I_{1.4 .9 .1}=O\left(\int_{1 / m}^{\delta} \int_{1 / n}^{\xi} \Phi(s, t)\left(\frac{A_{m,[1 / s]}}{s^{2}} \frac{B_{m,[1 / t]}}{t^{2}}\right) d t d s\right) \\
& =o\left(\int_{1 / m}^{\delta} \int_{1 / n}^{\xi}\left(\frac{\alpha\left(\frac{1}{s}\right) s}{\log \frac{1}{s}} \frac{\beta\left(\frac{1}{t}\right) t}{\log \frac{1}{t}}\right) \frac{A_{m,[1 / s]}}{s^{2}} \frac{B_{n,[1 / s]}}{t^{2}} d t d s\right) \\
& =o\left(\int_{1 / m}^{\delta} \int_{1 / n}^{\xi} \frac{\alpha\left(\frac{1}{s}\right)}{\log \frac{1}{s}} \frac{A_{m,[1 / s]}}{s} \frac{\beta\left(\frac{1}{t}\right)}{\log \frac{1}{t}} \frac{B_{n,[1 / s]}}{t} d t d s\right) \\
& =o\left(\int_{1 / \delta}^{m} \frac{\alpha(x) A_{m, x}}{x \log x} d x \int_{1 / \xi}^{n} \frac{\beta(y) B_{n, y}}{y \log y} d y\right)=o(1) \\
& I_{1.4 .9 .2}=O\left(\int_{1 / m}^{\delta} \int_{1 / n}^{\xi} \Phi(s, t) \frac{A_{m,[1 / s]}}{s^{2}} \frac{1}{t} \frac{d}{d t}\left(B_{n,[1 / t]}\right) d t d s\right) \\
& =O\left(\int_{1 / m}^{\delta} \int_{1 / n}^{\xi}\left(\frac{a\left(\frac{1}{s}\right) s}{\log \frac{1}{s}} \frac{\beta\left(\frac{1}{t}\right) t}{\log \frac{1}{t}}\right) \frac{A_{m,[1 / s]}}{s^{2}} \frac{1}{t} \frac{d}{d t}\left(B_{n,[1 / t]}\right) d t d s\right) \\
& =o\left(\int_{1 / m}^{\delta} \int_{1 / n}^{\xi} \frac{\alpha\left(\frac{1}{s}\right)}{\log \frac{1}{s}} \frac{A_{m,[1 / s]}}{s} \frac{\beta\left(\frac{1}{t}\right)}{\log \frac{1}{t}} \frac{d}{d t}\left(B_{n,[1 / t]}\right) d t d s\right) \\
& =o\left(\int_{1 / \delta}^{m} \frac{\alpha(x)}{\log x} \frac{A_{m, x}}{x} d x \int_{1 / n}^{\xi} \frac{\beta\left(\frac{1}{t}\right)}{\log \frac{1}{t}} \frac{d}{d t}\left(B_{n,[1 / t]}\right) d t d s\right) \\
& =o\left(\int_{1 / m}^{\delta} \frac{\alpha(x) A_{m, x}}{x \log x} d x\right) \int_{1 / n}^{\xi} \frac{\beta\left(\frac{1}{t}\right)}{\log \frac{1}{t}} \frac{d}{d t}\left(B_{n,[1 / t]}\right) d t d s \\
& =O(1) \int_{1 / n}^{\xi} O(1) \frac{d}{d t}\left(B_{n,[1 / t]}\right) d t, \quad \text { by the hypothesis of the theorem } \\
& =o(1)\left(B_{n,[1 / t]}\right)_{1 / n}^{\xi} \\
& =o(1), \quad a s,(m, n) \rightarrow \infty
\end{aligned}
$$

Similarly $I_{1.4 .9 .3}=o(1)$

$$
I_{1.4 .9 .3}=O\left(\int_{1 / m}^{\delta} \int_{1 / n}^{\xi} \Phi(s, t) \frac{1}{s} \frac{d}{d s}\left(A_{m,[1 / s]}\right)\right) \frac{1}{t} \frac{d}{d t}\left(B_{n,[1 / t]}\right) d t d s
$$




$$
\begin{aligned}
& =o\left(\int_{1 / m}^{\delta} \int_{1 / n}^{\xi}\left(\frac{\alpha\left(\frac{1}{s}\right) \beta\left(\frac{1}{t}\right) t}{\log \frac{1}{s} \log \frac{1}{t}}\right) \frac{1}{s} \frac{d}{d s}\left(A_{m,[1 / s]}\right)\right) \frac{1}{t} \frac{d}{d t}\left(B_{n,[1 / t]}\right) d t d s \\
& =o\left(\int_{1 / m}^{\delta} \int_{1 / n}^{\xi}\left(\frac{\alpha\left(\frac{1}{s}\right) \beta\left(\frac{1}{t}\right) t}{\log \frac{1}{s} \log \frac{1}{t}}\right) \frac{d}{d s}\left(A_{m,[1 / s]}\right)\right) \frac{d}{d t}\left(B_{n,[1 / t]}\right) d t d s \\
& =o(1) \int_{1 / m}^{\delta} \frac{\alpha\left(\frac{1}{s}\right)}{\log \frac{1}{s}} \frac{d}{d s}\left(A_{m,[1 / s]}\right) d s \int_{1 / n}^{\xi} \frac{\beta(1 / t)}{\log \frac{1}{t}} \frac{d}{d t}\left(B_{n,[1 / t]}\right) d t \\
& =o(1) \int_{1 / m}^{\delta} O(1) \frac{d}{d s}\left(A_{m,[1 / s]}\right) d s \int_{1 / n}^{\xi} O(1) \frac{d}{d t}\left(B_{n,[1 / t]}\right) d t d s,
\end{aligned}
$$

by the hypothesis of the theorem

$$
\begin{aligned}
& =o(1)\left[A_{m,[1 / s]}\right]_{1 / m}^{\delta}\left[B_{n,[1 / t]}\right]_{1 / n}^{\xi} \\
& =o(1), \text { as }(m, n) \rightarrow \infty .
\end{aligned}
$$

Thus,

$$
I_{1.4}=o(1), \text { as }(m, n) \rightarrow \infty .
$$

The above estimations, we get

$$
I_{1}=o(1) \text { as }(m, n) \rightarrow \infty
$$

Now, $m^{-1}<\delta<\pi, n^{-1}<\xi<\pi$. Then we obtain

$$
\begin{aligned}
I_{3} & \leq\left(\int_{\delta}^{\pi} \int_{0}^{\xi}\right)|\phi(s, t)| K_{m}(s) K_{n}(t) d s d t \\
& =\int_{\delta}^{\pi}\left|K_{m}(s)\right| d s \int_{0}^{1 / n}|\phi(s, t)|\left|K_{n}(t)\right| d t+\int_{\delta}^{\pi}\left|K_{m}(s)\right| d s \int_{1 / n}^{\xi}|\phi(s, t)|\left|K_{n}(t)\right| d t \\
& =I_{3.1}+I_{3.2}
\end{aligned}
$$

Taking

$$
\begin{aligned}
I_{3.1} & =\int_{\delta}^{\pi}\left|K_{m}(s)\right| d s \int_{0}^{1 / n}|\phi(s, t)|\left|K_{n}(t)\right| d t \\
& =O(n)\left(\int_{\delta}^{\pi}\left|K_{m}(s)\right| d s \int_{0}^{1 / n}|\phi(s, t)| d t\right) \quad \text { by Lemma (4.1) (ii) } \\
& =O(n) \int_{\delta}^{\pi} \frac{A_{m, \sigma}}{s} d s \int_{0}^{1 / n}|\phi(s, t)| d t \quad \text { by Lemma (4.3) } \\
& =O(n) \int_{0}^{\pi} \Phi_{2}\left(s, \frac{1}{n}\right) d s \\
& =O(n) o\left(\frac{\beta(n)}{n \log n}\right) \\
& =o\left(\frac{\beta(n)}{\log n}\right) \\
& =o(1), \text { as } n \rightarrow \infty .
\end{aligned}
$$


Further

$$
\begin{aligned}
I_{3.2} & =\int_{\delta}^{\pi}\left|K_{m}(s)\right| d s \int_{1 / n}^{\xi}|\phi(s, t)|\left|K_{n}(t)\right| d t \\
& =\left[\int_{\delta}^{\pi} \frac{A_{m, \sigma}}{s} d s \int_{1 / n}^{\xi} \phi(s, t) \frac{B_{n, \tau}}{t} d t\right] \text { by Lemma (4.3) and (4.4) } \\
& =O\left[\int_{\delta}^{\pi} d s\left\{\left[\Phi_{2}(s, t) \frac{B_{n, \tau}}{t}\right]_{1 / n}^{\xi}-\int_{1 / n}^{\xi} \Phi_{2}(s, t) \frac{d}{d t}\left(\frac{B_{n, \tau}}{t}\right) d t\right\}\right] \\
& =O\left[\int_{\delta}^{\pi} d s\left\{\Phi_{2}(s, \xi) \frac{B_{n,[1 / \xi]}}{\xi}-\Phi_{2}(s, 1 / n) n B_{n n}\right\}\right]+O\left[\int_{\delta}^{\pi} d s \int_{1 / n}^{\xi} \Phi_{2}(s, t) \frac{d}{d t}\left(\frac{B_{n, \tau}}{t}\right) d t\right] \\
& =O\left[\int_{\delta}^{\pi} \Phi_{2}(s, \xi) \frac{B_{n,[1 / \xi]}}{\xi} d s\right]+O(n) \int_{0}^{\pi} \Phi_{2}(s, 1 / n) d s+O\left[\int_{\delta}^{\pi} d s \int_{1 / n}^{\xi} \Phi_{2}(s, t) \frac{d}{d t}\left(\frac{B_{n, \tau}}{t}\right) d t\right] \\
& =O\left(\frac{B_{n,[1 / \xi]}}{\xi}\right) \int_{\delta}^{\pi} \Phi_{2}(s, \xi) d s+O(n) o\left(\frac{\beta_{(n)}}{n \log n}\right)+O\left[\int_{\delta}^{\pi} d s \int_{1 / n}^{\xi} \Phi_{2}(s, t) \frac{d}{d t}\left(\frac{\beta_{n, \tau}}{t}\right) d t\right] \\
& =o(1)+o(1)+o(1), \quad \text { similar to } I_{1.4 .9} \\
& =o(1), \quad \text { as }(m, n) \rightarrow \infty .
\end{aligned}
$$

Hence

$$
I_{3}=o(1), \text { as }(m, n) \rightarrow \infty
$$

Similarly we get

$$
I_{2}=o(1) \text { as }(m, n) \rightarrow \infty \text {. }
$$

By the regularity conditions of matrix summability and the Riemann-Lebesgue theorem we have

$$
I_{4}=o(1), \text { as }(m, n) \rightarrow \infty
$$

Therefore by the above estimations, our theorem is completely established.

\section{Particular Cases}

1. The result of Chow (1953) becomes a particular case of our theorem if $a_{m, i}=$ $\frac{1}{(m+1)} \forall i, b_{n, k}=\frac{1}{(n+1)} \forall k$ and $\alpha(u)=1, \forall u, \beta(v)=1 \forall v$.

2. If $a_{m, i}=\frac{1}{(m-i+1) \log m}, b_{n, k}=\frac{1}{(n-k+1) \log n}$ and $\alpha(u), \beta(v)$ are defined as in (1) then a result of Sharma (1958) becomes a particular case of our theorem.

3. A result of Tripathi and Singh (1981) becomes a particular case of our theorem if $a_{m, i}=\frac{P_{m-i}}{P_{m}}, b_{n, k}=\frac{q_{n-k}}{Q_{n}}$ provided $P_{m}=\sum_{i=0}^{n} p_{i}, Q_{n}=\sum_{k=0}^{n} q_{k}$ and $\alpha(u)=$ $\frac{u p(u) \log u}{P_{(u)}}$ and $\beta(v)=\frac{v q(v) \log v}{Q_{(v)}}$.

4. If $a_{m, i}, b_{n, k}$ are defined as in $(3)$ and $\alpha(u)=\frac{\log (u)}{P_{(u)}}, \beta(v)=\frac{\log (v)}{Q_{(v)}}$ then a result of Tripathi and Ojha (1982) becomes a particular case of our theorem. 
5. The result of Mishra (1985) becomes a particular case of our theorem if $a_{m, i}=\frac{p_{m-i}^{(1)}}{P_{m}^{(1)}}$, and $\alpha(u)=\frac{\log (u)}{\psi^{(1)}(u)}, \beta(v)=\frac{\log (v)}{\psi^{(2)}(v)}$.

6. If $a_{m, i}, b_{n, k}$ are defined as in (3) and $\alpha(u)=\frac{\log u \xi(u)}{\alpha P_{u}}, \beta(v)=\frac{\log v \psi(v)}{\alpha P_{v}}$ then a result of Lal (1992) becomes the particular case of our theorem.

7. The result of Singh, Lal and Singh (1994) becomes a particular case of our theorem if $a_{m, i}, b_{n, k}$ are defined in $(3)$ and $\alpha(u)=\frac{\xi(u) \log u}{P_{(u)}}, \quad \beta(v)=\frac{\psi(v) \log v}{Q_{(v)}}$.

\section{Acknowledgement}

Authors are grateful to Prof. L. M. Tripathi, Department of Maths, Banaras Hindu University, Varanasi-221005 (India) for suggesting the problems and to Prof. S. N. Lal. who has taken the pain to see the manuscripts of the paper. Authors are thankful to Prof. A. P. Dwivedi, Department of Maths and to Prof. V. K. Jain, Director, H. B. T. I., Kanpur for encouragement in this work. Shyam Lal one of the authors is grateful to the U.G.C., G.T. Road, Ghaziabad (U.P.) India, for providing financial assistance in the form of a minor research project vide letter no. F 3.3 (58)/1999-2000/MRP/NR dated 31.03.2000. The authors also express their gratitude to the referee for his valuable suggestions and comments for the improvement of third revised version of the paper.

\section{References}

[1] E. Hille and J. D. Tamarkin, On the summability of Fourier series, Trans. Amer. Math. Soc. 32(1932), 757-783.

[2] G. P. Singh, Shyam Lal and S. B. Singh, On $\left(N, p_{m}, q_{n}\right)$ summability of double Fourier series, Bul. Cal. Math. Soc. 87(1995), 151-156.

[3] H. Hirokawa, On the Nörlund summability of Fourier series and its conjugate series, Proc. Japan Acad. 44(1968), 449-451.

[4] J. G. Herriot, The Nörlund summability of double Fourier series, Trans. Amer. Math. Soc. 59(1942), 72-94.

[5] J. S. Siddigui, On harmonic summability of Fourier series, Proc. Nat. Acad. Sci. India Sect. A. 28(1948), 527-531.

[6] K. N. Mishra, Summability of double Fourier series by double Nörlund method, Bull Inst. Math. Acad. Sinica. 13(1985), 289-295.

[7] K. S. K. lyengar, A touberian theorem and its application to the convergence of a Fourier series, Proc. Indian Acad. Sci. 18-31.

[8] L. M. Tripathi and A. P. Singh, On Nörlund summability of the conjugate derived Fourier series, Journal of Scientific Research 31 (1981), 345-354.

[9] L. M. Tripathi and M. A. Ojha, A theorem on the Nörlund summability of the double Fourier series, Journal of Scientific Research 33 (1982), 245-251.

[10] L. M. Tripathi and Shyam Lal, A theorem on the Nörlund summability of a double Fourier series, Proc. Maths. 18(1984), 69-78. 
[11] P. L. Sharma, On the harmonic summability of double Fourier series, Proc. Amer. Math. Soc. 91(1958), 979-986.

[12] Shyam Lal, A study of double Fourier series by Nörlund summability, Acta Cincia Indica, Vol. XII M No.3(1992), 225-230.

[13] Shyam Lal and Sunita Verma, A theorem on $\left(N, p_{n}, q_{m}\right)$ summability of a double Fourier series, Journal of Scientific Research, B. H. U. 48(1998), 153-166.

[14] Shyam Lal and Ajay Pratap, On uniform matrix summability of a Fourier series, Bull. Ca. Math. Soc. 91(1999), 427-432.

[15] Shyam Lal, On the degree of approximation of conjugate of a function belonging to weighted $W\left[L^{p}, \xi(t)\right]$ class by matrix summability means of conjugate series of a Fourier series, Tamkang Journal of Mathematics 31(2000), 7-16.

[16] T. Pati, A generalization of a theorem of lyengar on the harmonic summability of Fourier series, Indian. J. Math. 3(1961), 85-90.

[17] T. Singh, On Nörlund summability of Fourier series and its conjugate series, Proc. Nat. Inst. Sci. India Part A. 29(1963), 65-73.

[18] Y. S. Chow, On the Cesàro summability of double Fourier series, Tôhoku Math. J. 5(1953), 277-283.

Department of Mathematics, Harcourt Butler Technological Institute, Nawabganj, Kanpur 208002, India.

Present address of Shyam Lal, Department of Mathematics, Faculty of Science University of Allahabad, Allahabad, 211002 (U.P.), India. 